\title{
Validación de la Escala CISNEROS de Medida de Mobbing al Gallego
}

\section{Validation of the Galician Version of the CISNEROS Mobbing Measurement Scale}

\author{
M. Ángeles López-Cabarcos, Paula Vázquez-Rodríguez y Carlos Montes-Piñeiro \\ Universidad de Santiago de Compostela
}

\begin{abstract}
Resumen. El objetivo del trabajo es la validación de la escala CISNEROS de medida de mobbing en gallego, formada por 43 ítems que miden la frecuencia de mobbing a través de una escala de 7 puntos, al tiempo que permite identificar a los posibles responsables del mismo. Para ello se llevó a cabo un análisis factorial exploratorio y un análisis factorial confirmatorio en una muestra multisectorial representativa de la población activa de Galicia. Los resultados del análisis factorial exploratorio indican la existencia de cinco factores principales que explican el $60,31 \%$ de la varianza total y muestran una elevada fiabilidad de la escala $(\alpha=.96)$. Posteriormente, se compara el ajuste de tres modelos mediante análisis factorial confirmatorio. Los resultados indican que el modelo de cinco factores correlacionados es el que mejor se ajusta a los datos, dando así apoyo empírico a la estructura de cinco factores obtenida en el análisis factorial exploratorio.
\end{abstract}

Palabras clave: mobbing, bullying, acoso psicológico, validez, análisis factorial.

\begin{abstract}
The aim of this work is the validation of the Galician version of the CISNEROS mobbing measurement scale, made up of 43 items that measure the mobbing frequency using a scale of 7 points, and at the same time allows the identification of the potential responsibles. So, we carried out a exploratory factor analysis and a confirmatory factor analysis in a multi-sectorial sample of the Galician active population. The results of the exploratory factor analysis indicate the existence of five main factors which explain $60.31 \%$ of the total variance and show a high reliability of the scale $(\alpha=.96)$. Subsequently, we compare the three models fit using confirmatory factor analysis. The results indicate that the correlated five-factor model provided the best fit to the data, giving empirical support to the five-factor structure obtained in the exploratory factor analysis.

Key words: mobbing, workplace bullying, psychological harassment, validity, factor analysis.
\end{abstract}

En los últimos años el acoso psicológico en el trabajo (mobbing) ha adquirido una relevancia importante debido a su consideración como riesgo psicosocial emergente, a su elevada incidencia entre la población trabajadora y a su potencialidad para causar numerosos e importantes daños sobre la víctima, la organización y la sociedad en general. Según datos oficiales, el mobbing afecta a un 5\% de los trabajadores europeos y a un $2.8 \%$ de los españoles (Parent-Thirion, FernándezMacías, Hurley y Vermeylen, 2007).

Se define como el intento repetido y sistemático de dañar a alguien por parte de un individuo o grupo de individuos, donde las víctimas tienen dificultad para defenderse y donde existe un desequilibrio de poder (real o percibido) entre las víctimas y sus acosadores

Este trabajo ha sido realizado al amparo de una ayuda para proyectos de investigación concedida en 2007 por la Vicepresidencia da Igualdade e do Benestar - Secretaría Xeral da Igualdade da Xunta de Galicia (VP438C 2007/17-0). La correspondencia de este artículo debe enviarse a la primera autora a la Universidad de Santiago de Compostela, Facultad de Administración y Dirección de Empresas, Campus de Lugo, Avda. Alfonso X el Sabio, s/n, 27002, Lugo. E-mail: angeles.lopez.cabarcos@usc.es
(Einarsen, 2000). Estos comportamientos negativos se traducen en actuaciones sutiles como el aislamiento o la exclusión del grupo, la minusvaloración del desempeño o la búsqueda de situaciones ridiculizantes. Leymann (1996) estableció cinco grupos diferentes de conductas que podían aparecer en un proceso de mobbing. El primer grupo incluye aquellas conductas dirigidas a reducir las posibilidades de comunicarse adecuadamente con otros, incluido el propio acosador. Se trata de impedir que la persona se pueda defender de los ataques sufridos (amenazas, críticas a la vida privada, etc.) o pueda expresarse libremente. El segundo grupo de conductas tiene por objeto evitar que la persona mantenga contactos sociales, bien aislándola físicamente o bien sometiéndola a un proceso de ninguneo. El tercer grupo de conductas persigue el descrédito o la humillación personal de la víctima que es objeto de burlas, críticas personales, ridiculizaciones o bromas pesadas. El cuarto grupo recoge aquellas conductas que suponen un ataque directo a la calidad profesional y a las posibilidades de desarrollo de la persona que las sufre. En este grupo se incluyen aspectos como la no asignación de tareas, o la asignación por encima o 
por debajo de la cualificación profesional. Por último, Leymann incluye un quinto grupo cuyo objetivo es atentar contra la salud de la persona, asignándole tareas peligrosas, impidiéndole que tome las medidas de seguridad oportunas para la realización del trabajo o, incluso, sometiéndole a abusos físicos o sexuales.

Desde los años ochenta numerosos autores se han implicado en el desarrollo de técnicas, tanto cualitativas como cuantitativas, para el análisis y medida del mobbing. Las primeras investigaciones sobre el tema se realizaron a través de casos clínicos y se centraron en la descripción de los hechos y en sus consecuencias (Moreno-Jiménez, Rodríguez-Muñoz, MartínezGamarra y Gálvez, 2007). Posteriormente, y dentro de esta perspectiva cualitativa, se desarrollaron otras técnicas, tales como las entrevistas personales (Archer, 1999; Coyne, Seigne y Randall, 2000), el registro diario de los hechos (Gross, 2002), los estudios de caso (Matthiesen, Aasen, Holst, Wie y Einarsen, 2003), los focus group (Liefooghe y Mackenzie Davey, 2001, 2003) o la técnica de los incidentes críticos (Liefooghe y Olafsson, 1999). Sin embargo, la técnica que ha adquirido mayor relevancia en el estudio del acoso psicológico en las organizaciones es el cuestionario (Einarsen y Raknes, 1997; Leymann, 1990).

En su forma más simple el cuestionario puede incluir una única pregunta en la que se le pida al encuestado que manifieste de forma afirmativa o negativa si ha sido víctima de un proceso de mobbing. Algunos investigadores van más allá e incluyen una definición de mobbing acompañada de una cuestión en la que se le pide al sujeto encuestado que indique la duración y la frecuencia con la que ha sufrido el proce- so descrito. Este método subjetivo de autodiagnóstico ha sido complementado (y en algún caso sustituido) con un método denominado operativo consistente en presentar al trabajador un listado de conductas características de mobbing y pedirle que señale la frecuencia con que las ha sufrido durante un período de tiempo determinado (Einarsen, 2000). Se considera que este método de medida es objetivo, si bien es cierto que el nivel de objetividad, aunque mayor que en los métodos basados en la definición, es relativo puesto que de nuevo es la víctima quien determina qué conductas ha sufrido y con qué frecuencia y duración. Por ello, Agervold (2007) define este tipo de métodos como cuasi-objetivos.

Este segundo método ha llevado a numerosos autores a desarrollar escalas de medida de mobbing, tales como el Leymann Inventory of Psychological Terrorization (LIPT) (Leymann, 1990), el Negative Acts Questionnaire (NAQ) (Einarsen y Raknes, 1997), la Work Harassment Scale (WHS) (Björkqvist, Österman y Hjelt-Bäck, 1994), el Bergen Bullying Index (Einarsen et al., 1994), la escala LIPT reducida de Van Dick y Wagner (2001), la Workplace Incivility Scale (WIS) (Cortina, Magley, Williams y Langhout, 2001), el Generalized Workplace Harassment Questionnaire (GWHQ) (Rospenda y Richman, 2004) y el Uncivil Workplace Behaviour Questionnarie (UWBQ) (Martin y Hine, 2005); siendo las más conocidas y utilizadas el LIPT y el NAQ. Ambos cuestionarios han sido empleados en numerosas investigaciones en diferentes sectores de actividad, y han sido traducidos y adaptados a varios idiomas, surgiendo en ocasiones diferentes estructuras factoriales (ver Tabla 1).

Tabla 1. Estructura factorial del LIPT y del NAQ

\begin{tabular}{llll}
\hline Autores & $\begin{array}{c}\text { Escala } \\
\text { (Item) }\end{array}$ & $\begin{array}{c}\text { Varianza } \\
\text { Explicada }\end{array}$ & Factores \\
\hline
\end{tabular}

Leymann (1990)

LIPT (45)

Niedl (1995)

LIPT (45)

$36.5 \%$

$8.6 \%$

$4.8 \%$

$4.5 \%$

$4.0 \%$

$3.4 \%$

$2.9 \%$

Zapf, Knorz y Kulla (1996)
LIPT (45)

\author{
Formas de comunicación \\ Aislamiento \\ Descrédito profesional \\ Desprestigio personal \\ Amenazas de violencia física
}

Ataques a la integridad personal (13

ítems; $\alpha=.93$ )

Aislamiento (11 ítems; $\alpha=.91)$

Crítica directa e indirecta ( 5 ítems; $\alpha=.83$ )

Sanciones a través de tareas ( 5 ítems; $\alpha=.81$ )

Amenazas ( 3 ítems; $\alpha=.63$ )

Acoso sexual ( 3 ítems; $\alpha=.71$ )

Ataques a la vida privada (4 ítems; $\alpha=.71$ )

Medidas organizacionales (11 ítems; $\alpha=.86$ )

Aislamiento social ( 6 ítems; $\alpha=.83$ )

Ataques a la vida privada ( 7 ítems; $\alpha=.75$ )

(Continúa en pág. siguiente) 
Tabla 1. Estructura factorial del LIPT y del NAQ (continuación)

\begin{tabular}{|c|c|c|c|}
\hline Autores & $\begin{array}{l}\text { Escala } \\
\text { (Item) }\end{array}$ & $\begin{array}{l}\text { Varianza } \\
\text { Explicada }\end{array}$ & Factores \\
\hline & & $\begin{array}{l}- \\
- \\
- \\
-\end{array}$ & $\begin{array}{l}\text { Violencia física (6 ítems; } \alpha=.69 \text { ) } \\
\text { Ataque a las actitudes de la víctima ( } 3 \text { ítems; } \alpha=.63 \text { ) } \\
\text { Agresión verbal ( } 3 \text { ítems; } \alpha=.66 \text { ) } \\
\text { Rumores ( } 2 \text { ítems; } \alpha=.65 \text { ) }\end{array}$ \\
\hline Zapf (1999) & LIPT (45) & $\begin{array}{l}- \\
- \\
- \\
- \\
- \\
-\end{array}$ & $\begin{array}{l}\text { Medidas organizacionales ( } 13 \text { ítems; } \alpha=.92 \text { ) } \\
\text { Aislamiento social ( } 4 \text { ítems; } \alpha=.81 \text { ) } \\
\text { Ataques a la vida privada ( } 5 \text { ítems; } \alpha=.74 \text { ) } \\
\text { Violencia física ( } 4 \text { ítems; } \alpha=.54 \text { ) } \\
\text { Agresión verbal ( } 6 \text { ítems; } \alpha=.85 \text { ) } \\
\text { Rumores ( } 2 \text { ítems; } \alpha=.70 \text { ) }\end{array}$ \\
\hline Einarsen y Raknes (1997) & NAQ (22) & $\begin{array}{l}31.7 \% \\
- \\
-\end{array}$ & $\begin{array}{l}\text { Ataques personales ( } 9 \text { ítems; } \alpha=.85 \text { ) } \\
\text { Acoso relacionado con el trabajo ( } 3 \text { ítems; } \alpha=.57 \text { ) } \\
\text { Exclusión social ( } 3 \text { ítems; } \alpha=.33 \text { ) }\end{array}$ \\
\hline Einarsen y Hoel (2001) & NAQ (29) & $\begin{array}{l}- \\
-\end{array}$ & $\begin{array}{l}\text { Acoso personal ( } 12 \text { ítems; } \alpha=.87 \text { ) } \\
\text { Acoso relacionado con el trabajo ( } 8 \text { ítems; } \alpha=.81 \text { ) }\end{array}$ \\
\hline Einarsen y Hoel (2006) & NAQ (22) & $\begin{array}{l}- \\
-\end{array}$ & $\begin{array}{l}\text { NAQ-person (14 ítems; } \alpha=.86 \text { ) } \\
\text { NAQ-tasks ( } 8 \text { ítems; } \alpha=.76 \text { ) }\end{array}$ \\
\hline García-Izquierdo et al. (2004) & NAQ (24) & $66.7 \%$ & $\begin{array}{l}\text { Conductas hostiles que afectan al entorno } \\
\text { social ( } 9 \text { ítems; } \alpha=.88 \text { ) } \\
\text { Conductas hostiles relacionadas con el } \\
\text { desempeño del trabajo ( } 6 \text { ítem; } \alpha=.82 \text { ) } \\
\text { Conductas discriminatorias asociadas al } \\
\text { género y alaedad ( } 6 \text { ítems; } \alpha=.72 \text { ) } \\
\text { Amenazas o abusos físicos, insultos e } \\
\text { indirectas paraabandonar el trabajo } \\
\text { (3 ítem; } \alpha=.79 \text { ) }\end{array}$ \\
\hline $\begin{array}{l}\text { Meseguer, Soler, Sáez } \\
\text { y García-Izquierdo (2007) }\end{array}$ & NAQ (24) & $54.0 \%$ & $\begin{array}{l}\text { Acoso personal ( } 8 \text { ítems) } \\
\text { Acoso centrado en el rendimiento del trabajo ( } 5 \text { ítems) } \\
\text { Acoso por razones de edad y género ( } 5 \text { ítems) } \\
\text { Acoso organizativo ( } 3 \text { ítems) } \\
\text { Otras formas de acoso ( } 3 \text { ítems) }\end{array}$ \\
\hline Moreno-Jiménez et al. (2007) & NAQ (14) & $63.4 \%$ & $\begin{array}{l}\text { Acoso relacionado con el trabajo (8 ítems; } \\
\alpha=.79 \text { muestrade víctimas y } \alpha=.84 \\
\text { muestrano-víctimas) } \\
\text { Acoso personal ( } 6 \text { ítems; } \alpha=.84 \text { muestra } \\
\text { de víctimas y } \alpha=.82 \text { muestra no-víctimas) }\end{array}$ \\
\hline
\end{tabular}

En España, a diferencia de otros países donde se ha normalizado el empleo de uno o dos instrumentos para medir la incidencia del mobbing, se emplean múltiples herramientas en el análisis de este tipo de procesos, lo que provoca serias dificultades para comparar los resultados de los diferentes estudios. Además de las adaptaciones que se han realizado del LIPT (González de Rivera y Rodríguez-Abuín, 2003) y del NAQ (García-Izquierdo et al., 2004; Meseguer et al., 2007;
Moreno-Jiménez et al., 2007), algunos investigadores han desarrollado sus propias escalas de medida de mobbing. Es el caso de la escala CISNEROS (Fidaldo y Piñuel, 2004), las escalas elaboradas por Boada, De Diego y Vigil (2003), el Cuestionario de Hostigamiento Psicológico en el Trabajo (HPT) (Fornés, Martínez-Abascal y García de la Banda, 2008) o el Cuestionario de Acoso Psicológico en el Trabajo (CAPT) (Moreno-Jiménez et al., 2008). 
Precisamente, la escala CISNEROS fue la primera herramienta que se empleó en España para medir la incidencia del mobbing y forma parte del Barómetro Cisneros (Cuestionario Individual sobre Psicoterror, Negación, Estigmatización y Rechazo en Organizaciones Sociales), instrumento que se utiliza para sondear de manera periódica el estado y los índices de la violencia en el entorno laboral de las organizaciones (Piñuel y Oñate, 2006).

La escala CISNEROS, específicamente diseñada para evaluar las conductas de mobbing, su frecuencia y su duración, sigue las mismas pautas que el LIPT de Leymann y objetiva 43 conductas de acoso psicológico en el trabajo, solicitando al encuestado que valore de 0 (nunca) a 6 (todos los días) el grado en que es sometido a cada una de las conductas descritas (Piñuel y Oñate, 2006). Al igual que el LIPT-60 (González de Rivera y Rodríguez-Abuín, 2003), la corrección de la escala se lleva a cabo mediante el número total de estrategias de acoso (NEAP), el índice global de acoso psicológico (IGAP) y el índice medio de intensidad de las estrategias de acoso (IMAP). Los dos últimos índices deben considerarse de forma conjunta para entender correctamente su significado. El valor del IMAP es siempre mayor o igual que el del IGAP, ya que el primero divide el sumatorio de la puntuación entre el número de ítems con respuesta positiva, en tanto el segundo divide siempre por el número total de ítems (43). De esta forma, cuando existen diferencias muy grandes entre el IMAP y el IGAP se entiende que el sujeto sufre pocas conductas de acoso psicológico, pero con una elevada intensidad (Fidalgo y Piñuel, 2004)

Los análisis psicométricos llevados a cabo por Fidalgo y Piñuel (2004) muestran una alta fiabilidad de la escala, ofreciendo valores indicativos de una alta consistencia interna entre los ítems que componen la escala (método de las dos mitades: .96; método alfa de Cronbach: .97), además de una buena capacidad discriminatoria. En cuanto a la validez de contenido, los autores consideran el acuerdo que existe entre las conductas objetivadas en la escala y las recogidas en otros instrumentos de medida de mobbing como un primer indicador de su validez. Adicionalmente, siguiendo el método del escalamiento multidimensional, dividen las conductas en dos dimensiones: ámbito de la conducta de acoso psicológico (profesional vs. personal) y naturaleza de la conducta (coercitiva/punitiva vs. ninguneo/humillación). La validez de contenido se ve apoyada al comprobar que, al igual que se recoge en la literatura, las formas de acoso psicológico más frecuentes se corresponden con conductas de ninguneo relacionadas con el ámbito profesional. Sobre la validez de criterio, la escala muestra correlaciones significativas con el síndrome de estrés postraumático, con síntomas de depresión, con la intención de abandonar la organización, y con dos de las dimensiones del burnout-agotamiento emocional y despersonalización-.
Teniendo en cuenta que: i) la escala CISNEROS es el primer instrumento de medida del mobbing que ha sido diseñado acorde a las particularidades del ambiente de trabajo español y en idioma castellano, incluyendo una mayor diversidad de conductas que otras escalas de medida; ii) ha sido empleada en el análisis de la incidencia del mobbing en España en numerosas ocasiones, tanto a nivel sectorial como multisectorial, facilitándose así la comparación de los datos; iii) es una de las escalas más reconocidas a nivel divulgativo, no en vano su autor fue uno de los pioneros en el estudio del mobbing en nuestro país, y iv) no existe ningún instrumento traducido al gallego y validado para Galicia; el objetivo de este estudio instrumental (Montero y León, 2007) es validar la escala CISNEROS en la población gallega, analizando la consistencia interna y la estructura factorial de la misma.

\section{Método}

\section{Participantes}

Se ha empleado una muestra de 384 trabajadores mayores de 18 años pertenecientes a la comunidad autónoma gallega -representativa de la población activa $(e= \pm 5.0 \%)$-, de los cuales 218 fueron hombres $(56.8 \%)$ y 166 fueron mujeres $(43.2 \%)$. Todos ellos con una edad media de 33.21 años $(D T=10.62)$. Las características demográficas y laborales más relevantes se detallan en la Tabla 2.

\section{Instrumento}

La escala Cisneros (Fidalgo y Piñuel, 2004) objetiva 43 conductas de mobbing (por ejemplo, "Evalúan mi trabajo de manera inequitativa o de forma sesgada", "Prohíben a mis compañeros o colegas hablar conmigo") ${ }^{1}$ en las que las personas señalan la frecuencia con la que sufren cada una de ellas, en una escala que va de 0 (nunca) a 6 (todos los días). Asimismo, permite identificar la procedencia del acoso para cada conducta, distinguiendo entre acoso psicológico procedente de jefes, compañeros o subordinados. Ofrece tres índices de acoso: el número total de estrategias de acoso (NEAP), el índice global de acoso psicológico (IGAP) y el índice medio de la intensidad de las estrategias de acoso (IMAP). Además, la escala incluye un ítem adicional de respuesta dicotómica sí/no (ítem 44) que recoge la percepción subjetiva del sujeto para calificar un caso como mobbing: "En el transcurso de los últimos 6 meses, ¿ha sido Ud víctima de por lo menos alguna de las anteriores formas de maltrato psicológico de manera continuada (con una frecuencia de más

\footnotetext{
${ }^{1}$ La versión en gallego de la escala CISNEROS puede solicitarse al primer autor.
} 
Tabla 2. Caracterización sociodemográfica y laboral de la muestra

\begin{tabular}{|c|c|c|c|}
\hline & Hombres & Mujeres & Total \\
\hline \multicolumn{4}{|l|}{ Estado civil } \\
\hline Soltero & $58.8 \%$ & $58.4 \%$ & $58.6 \%$ \\
\hline Casado & $32.4 \%$ & $31.3 \%$ & $31.9 \%$ \\
\hline Pareja de hecho & $5.6 \%$ & $3.0 \%$ & $4.5 \%$ \\
\hline Separado-divorciado & $2.8 \%$ & $5.4 \%$ & $3.9 \%$ \\
\hline Viudo & $0.5 \%$ & $1.8 \%$ & $1.0 \%$ \\
\hline \multicolumn{4}{|l|}{ Estudios } \\
\hline Primaria & $23.0 \%$ & $12.7 \%$ & $18.6 \%$ \\
\hline Bachillerato & $19.8 \%$ & $20.6 \%$ & $20.2 \%$ \\
\hline Formación profesional & $26.7 \%$ & $19.4 \%$ & $23.6 \%$ \\
\hline Titulación superior & $26.3 \%$ & $45.5 \%$ & $34.6 \%$ \\
\hline Doctorado & $2.3 \%$ & $1.8 \%$ & $2.1 \%$ \\
\hline Otros & $1.8 \%$ & - & $1.1 \%$ \\
\hline \multicolumn{4}{|l|}{ Sector de actividad } \\
\hline Agricultura, ganadería, caza y silvicultura & $2.8 \%$ & $0.6 \%$ & $1.9 \%$ \\
\hline Pesca & $1.9 \%$ & $0.6 \%$ & $1.3 \%$ \\
\hline Industria extractiva & $1.4 \%$ & $1.9 \%$ & $1.6 \%$ \\
\hline Industria manufacturera & $12.0 \%$ & $5.7 \%$ & $9.3 \%$ \\
\hline \multicolumn{3}{|l|}{ Producción y distribución de energía eléctrica, } & $3.4 \%$ \\
\hline Construcción & $13.0 \%$ & $1.3 \%$ & $7.9 \%$ \\
\hline Comercio, reparación de vehículos de motor & $12.5 \%$ & $22.0 \%$ & $16.6 \%$ \\
\hline Hostelería & $7.9 \%$ & $13.8 \%$ & $10.5 \%$ \\
\hline Trasporte, almacenamiento y comunicaciones & $6.0 \%$ & $5.0 \%$ & $5.6 \%$ \\
\hline Intermediación financiera & $3.7 \%$ & $2.5 \%$ & $3.2 \%$ \\
\hline $\begin{array}{l}\text { Actividades inmobiliarias y de alquiler, } \\
\text { servicios empresariales }\end{array}$ & $6.5 \%$ & $4.4 \%$ & $5.6 \%$ \\
\hline \multicolumn{4}{|l|}{ Administración pública, defensa y seguridad } \\
\hline social obligatoria & $9.3 \%$ & $7.6 \%$ & $8.5 \%$ \\
\hline Educación & $8.3 \%$ & $13.8 \%$ & $10.7 \%$ \\
\hline Actividades sanitarias y veterinarias, servicios sociales & $1.9 \%$ & $10.1 \%$ & $5.4 \%$ \\
\hline \multicolumn{4}{|l|}{ Otras actividades sociales y de servicios prestados } \\
\hline a la comunidad & $6.5 \%$ & $8.8 \%$ & $7.5 \%$ \\
\hline Actividad del hogar & - & $1.9 \%$ & $0.8 \%$ \\
\hline Organismos extraterritoriales & $0.5 \%$ & - & $0.3 \%$ \\
\hline \multicolumn{4}{|l|}{ Situación laboral } \\
\hline Eventual & $29.1 \%$ & $41.7 \%$ & $34.6 \%$ \\
\hline Fijo & $59.6 \%$ & $53.4 \%$ & $56.9 \%$ \\
\hline Otros & $11.3 \%$ & $4.9 \%$ & $8.5 \%$ \\
\hline
\end{tabular}

de 1 vez por semana)?". Con respecto a las propiedades psicométricas de la escala original cabe señalar que Fidalgo y Piñuel hallaron una fiabilidad (alfa de Cronbach) de .97.

\section{Procedimiento}

Siguiendo la guía propuesta por Carretero-Dios y Pérez (2005), hemos desarrollado la adaptación de la escala Cisneros al gallego en 3 fases: (1) traducción, (2) prueba piloto, y (3) validación.

El primer paso consistió en la traducción de la escala al gallego, para lo que se contó con el asesoramiento del Servicio de Normalización Lingüística de la Univer- sidad de Santiago de Compostela. Una vez traducida al gallego, la escala fue traducida de nuevo al castellano para comprobar que se mantenía el significado original de cada ítem. Este procedimiento de traducción converge con el marco establecido por Brislin (1986) en lo referente a la equivalencia de las traducciones.

El siguiente paso consistió en la realización de una prueba piloto para comprobar la comprensión lingüística y de idioma del cuestionario. La prueba se realizó entre una muestra formada por estudiantes de diferentes campus universitarios de Galicia, con el fin de testar la comprensión en áreas geográficas con registros de gallego muy diferentes. En un primer momento se pasó el cuestionario a un total de 72 estudiantes, a los que se les pidió que subrayaran aquellas palabras que 
no conocían o aquellos ítems que no entendían. Tras la recopilación de datos inicial se procedió a corregir, eliminar o sustituir aquellas palabras que, siendo del idioma gallego, fueran señaladas por más de un $5 \%$ de personas. Corregido el cuestionario, se procedió a realizar un segundo pase entre una muestra de estudiantes de la Facultad de Administración y Dirección de Empresas de la Universidad de Santiago de Compostela en el campus de Lugo. En este caso no se realizó ninguna corrección en el cuestionario.

Por último, el cuestionario fue administrado durante los meses de septiembre y octubre de 2007 a la muestra escogida, previa obtención del consentimiento verbal de los interesados en participar en el estudio, con el objetivo de validar la prueba en la población gallega y cuyos resultados se recogen en el presente trabajo. El análisis de los datos se realizó mediante el paquete estadístico SPSS 15.0.

\section{Resultados}

Antes de realizar el análisis factorial se consideraron diferentes criterios para valorar la viabilidad del mismo: el determinante de la matriz de correlaciones presentó un valor $<.001$, la prueba de esfericidad de Bartlett fue significativa, y el test KMO de adecuación de la muestra alcanzó un valor $>.80$. Estos valores indican la pertinencia del análisis factorial (Hair, Anderson, Tatham y Black, 1999).

Posteriormente, las repuestas de los sujetos a los ítems de la escala Cisneros fueron sometidas a un análisis factorial de componentes principales con rotación Varimax. Como resultado se obtuvieron 9 factores con eigenvalue o autovalor superior a 1.00 (Kaiser, 1960), que explican el $72.03 \%$ de la varianza total. Todos los ítems del cuestionario, excepto el ítem 4 ("Me fuerzan a realizar trabajos que van contra mis principios o mi

Tabla 3. Estructura factorial de la versión en gallego de la escala Cisneros

\begin{tabular}{|c|c|c|c|c|c|}
\hline Ítem - Contenido & $\mathrm{F} 1$ & $\mathrm{~F} 2$ & $\mathrm{~F} 3$ & F4 & F5 \\
\hline 35. Perjuicio imagen & .84 & .12 & .10 & .10 & .01 \\
\hline 20. Amenazas uso disciplinario & .81 & .14 & -.03 & -.03 & .10 \\
\hline 27. Amenazas en persona & .73 & .01 & .24 & .05 & .01 \\
\hline 22. Distorsión comunicación & .72 & .23 & .27 & .27 & .28 \\
\hline 23. Desestabilización & .69 & .19 & .25 & .15 & .19 \\
\hline 24. Menosprecio & .69 & .14 & .46 & .11 & .21 \\
\hline 19. Humillaciones & .68 & .24 & .32 & .25 & .35 \\
\hline 29. Gritos & .61 & .34 & .29 & .37 & .18 \\
\hline 40. Desmoralizar & .61 & .31 & .24 & .45 & .12 \\
\hline 32. Rumores y calumnias & .58 & .35 & .17 & .41 & .01 \\
\hline 18. Amplificación errores & .58 & .46 & .12 & .10 & .12 \\
\hline 17. Acusación sistemática & .57 & .41 & .25 & .31 & .28 \\
\hline 41. Inducir a errores & .54 & .29 & .30 & .40 & -.08 \\
\hline 21. Medidas de aislamiento & .53 & -.01 & .11 & .43 & .01 \\
\hline 42. Monitorización perversa & .46 & .27 & .39 & .21 & -.05 \\
\hline 15. Minusvaloración desempeño & .46 & .42 & .21 & .42 & .21 \\
\hline 05. Evaluación no equitativa & .46 & .29 & .16 & .28 & .42 \\
\hline 37. Reducción de plazos & .20 & .79 & .05 & .25 & .05 \\
\hline 36. Presión indebida & .37 & .75 & .07 & .28 & .16 \\
\hline 38. Modificación de responsabilidad & .06 & .73 & .34 & .14 & .04 \\
\hline 39. Infravaloración esfuerzo & .42 & .68 & .16 & .26 & .12 \\
\hline 09. Tareas monótonas & .26 & .57 & -.04 & -.13 & .16 \\
\hline 08. Tareas por debajo de competencia & .07 & .42 & .08 & .14 & .37 \\
\hline 33. Privar de información & .24 & .41 & .24 & .18 & .30 \\
\hline 26. Críticas vida personal & .23 & .07 & .83 & .10 & .02 \\
\hline 31. Ridiculización & .24 & .15 & .78 & .04 & .05 \\
\hline 25. Burlas & .32 & .19 & .74 & .03 & -.04 \\
\hline 30. Avasallamiento físico & .18 & .05 & .66 & -.05 & .27 \\
\hline 11. Tareas peligrosas & .16 & .13 & -.08 & .78 & .02 \\
\hline 12. Impedir seguridad & .19 & .16 & -.03 & .72 & .33 \\
\hline 10. Sobrecarga & .21 & .41 & .16 & .63 & .09 \\
\hline 34. Limitación de carrera profesional & .36 & .33 & .09 & .59 & .28 \\
\hline 02. Ignorar & .27 & .11 & -.06 & .00 & .76 \\
\hline 03. Interrupciones continuas & .14 & .17 & .30 & .22 & .68 \\
\hline 01. Restricción comunicación & -.01 & .06 & .05 & .23 & .67 \\
\hline Varianza explicada (\%) & 40.02 & 6.56 & 5.63 & 4.10 & 3.99 \\
\hline
\end{tabular}

Nota. En negrita se muestra el factor al que ha sido asignado cada ítem. F1: Degradación personal; F2: Degradación profesional; F3: Humillación personal; F4: Exposición a riegos de modo deliberado; F5: Ninguneo. 
ética"), saturaron igual o superior a .40 en al menos un factor (Nunnally, 1978), aunque muchos de ellos presentan cargas factoriales en más de un factor. En este caso, se asignó el ítem al factor en que presentaba mayor saturación.

En la Tabla 3 se muestran las saturaciones factoriales de los cinco primeros factores que, en conjunto, explican el $60.31 \%$ de la varianza total de la prueba. Se les ha dado nombre en función del contenido predominante de los ítems que los componen y de la literatura existente. Los cuatro últimos factores no se incluyen al haber sido considerados residuales por contener sólo uno o dos ítems y explicar un bajo porcentaje de varianza.

Atendiendo a estas consideraciones y tomando nuevamente una saturación mayor o igual a .40 como referencia para la interpretación de los factores podemos señalar lo siguiente:

- Factor 1: Explica por sí solo el $40.02 \%$ de la varianza. Está constituido por 17 ítems con saturaciones que van de .46 a .84 y que engloban un
26), ridiculización (ítem 31), burlas (ítem 25), llegando incluso al avasallamiento físico (ítem 30).

- Factor 4: Constituido por 4 ítems con saturaciones entre .59 y .78 . Este factor explica el $4.10 \%$ de la varianza y su contenido alude a conductas deliberadas y malintencionadas como la asignación de tareas peligrosas (ítem 11), impedir el uso de medidas de seguridad (ítem 12), sobrecargar de trabajo (ítem 10) y limitar la carrera profesional (ítem, 34). Se ha denominado este factor como Exposición a riesgos de modo deliberado.

- Factor 5: Está constituido por 3 ítems que explican el $3.99 \%$ de la varianza, con saturaciones superiores a .67. El contenido de estos ítems hace referencia a conductas como ignorar (ítem 2), interrumpir continuamente (ítem 3 ) y restringir la comunicación (ítem 1). Este factor se ha denominado Ninguneo.

En la Tabla 4 se presentan los estadísticos descriptivos, fiabilidad y correlaciones correspondientes a los cinco factores que integran la escala para el total de la

Tabla 4. Estadísticos descriptivos, correlaciones y fiabilidad de los factores de la versión en gallego de la escala Cisneros

\begin{tabular}{|c|c|c|c|c|c|c|c|c|c|}
\hline & Media & $D T$ & Min. & Máx. & $\mathrm{F} 1$ & $\mathrm{~F} 2$ & F3 & $\mathrm{F} 4$ & F5 \\
\hline $\mathrm{F} 1$ & 0.24 & 0.64 & 0 & 5.94 & $(.96)$ & & & & \\
\hline $\mathrm{F} 2$ & 0.47 & 0.84 & 0 & 5.00 & $.70^{*}$ & $(.86)$ & & & \\
\hline F3 & 0.08 & 0.37 & 0 & 4.00 & $.59 *$ & $.41^{*}$ & $(.84)$ & & \\
\hline F4 & 0.21 & 0.64 & 0 & 6.00 & $.64 *$ & $.60 *$ & $.25^{*}$ & $(.84)$ & \\
\hline F5 & 0.37 & 0.80 & 0 & 6.00 & $.47 *$ & $.47 *$ & $.29 *$ & $.42 *$ & $(.75)$ \\
\hline
\end{tabular}

Nota: * p<.01. Entre paréntesis se muestra la consistencia interna de los factores F1: Degradación personal; F2: Degradación profesional; F3: Humillación personal; F4: Exposición a riegos de modo deliberado; F5: Ninguneo.

amplio espectro de conductas de acoso como críticas y reproches (ítems 17,18 y 32 ), minusvaloración (ítems 15, 19, 24 y 40), provocaciones para forzar errores (ítems 23, 42 y 41), amenazas (ítems 20 y 27), gritos (ítem 29), discriminaciones (ítem 5) y otras medidas que pretenden dañar la imagen (ítem 35), distorsionar la comunicación (ítem 22) y aislar socialmente al trabajador (ítem 21). Se ha denominado Degradación personal.

- Factor 2: Lo constituyen 7 ítems con saturaciones comprendidas entre .41 y .79 . Explican el $6.56 \%$ de la varianza. Su contenido alude a comportamientos orientados a la limitación profesional mediante la reducción de plazos (ítem 37), presión indebida (ítem 36), trato inferior al grado de capacitación (ítems 38, 8 y 9) infravaloración del esfuerzo (ítem 39) y privar información (ítem 33). Este factor se ha denominado Degradación profesional.

- Factor 3: Lo constituyen 4 ítems que explican el $5.63 \%$ de la varianza. Todas las saturaciones son superiores a .66 y su contenido se refiere a conductas orientadas a la Humillación personal como continuas críticas a la vida personal (ítem muestra. La fiabilidad de los factores es satisfactoria, con coeficientes alfa que varían de .75 (Ninguneo) a .96 (Degradación personal), alcanzando un valor de .96 para el total de la escala. Asimismo, las correlaciones entre los factores -obtenidas a partir de las puntuaciones factoriales de los sujetos- son elevadas, oscilando entre .25 y .70 .

A pesar de que el análisis factorial exploratorio revela una estructura multidimensional, el análisis minucioso de los factores extraídos sugiere que puede tratarse de un cuestionario unidimensional. Esto se debe fundamentalmente a que el primer factor (degradación personal) aglutina el mayor número de ítems de las escala (17), abarcando por ello una gran variedad de comportamientos relacionados con el mobbing, y explica por sí solo un $40 \%$ de la varianza total del cuestionario ${ }^{2}$. Así, para clarificar la estructura factorial de la escala, se llevó a cabo un análisis factorial confirmatorio con el objetivo de comparar tres modelos diferentes (un factor, cinco factores independientes y cinco factores correlacionados). Los análisis se llevaron a

\footnotetext{
${ }^{2}$ Agradecemos al revisor esta observación y las sugerencias para su
} solución. 
cabo mediante el programa estadístico AMOS 6.0 (Arbuckle, 2005) y se emplearon diferentes índices para evaluar el ajuste de los modelos: chi-cuadrado dividido por los grados de libertad $\left(\chi^{2} / \mathrm{gl}\right)$, el índice de bondad de ajuste (Goodness of Fit Index, GFI), el índice de ajuste comparativo (Comparative Fit Index, CFI), la raíz cuadrada media residual (Root Mean Square Residual, RMR,) y la raíz cuadrada media del error de aproximación (Root Mean Square Error of Approximation, RMSEA). Estos índices son algunos de los más empleados debido a que son menos sensibles al tamaño muestral (García, Gallo y Miranda, 1998). La Tabla 5 resume el ajuste de los tres modelos comparados. le tareas que exceden sus capacidades u obligaciones laborales, o bien ordenándole la ejecución de tareas rutinarias o por debajo de su capacidad profesional. El objetivo en este caso es llamar la atención sobre la incapacidad profesional de la víctima. El tercer factor, humillación personal, aglutina aquellos comportamientos que buscan humillar a la víctima aludiendo aspectos de su vida personal y burlándose de su aspecto físico o su forma de ser, llegando incluso a la agresión física. El cuarto factor, exposición a riesgos de modo deliberado, incluye comportamientos que indican una exposición de forma malintencionada a riesgos de carácter físico y psicosocial, impidiendo al trabajador que adopte las medidas de seguridad necesarias

Tabla 5. Índices de bondad de ajuste para cada uno de los modelos propuestos

\begin{tabular}{|c|c|c|c|c|c|}
\hline & $\chi^{2 / g 1}$ & CFI & GFI & RMR & RMSEA \\
\hline Modelo de un factor & 7.09 & .78 & .67 & .06 & .13 \\
\hline Modelo de cinco factores independientes & 4.84 & .86 & .78 & .06 & .10 \\
\hline Modelo de cinco factores correlacionados & 2.59 & .95 & .88 & .04 & .06 \\
\hline
\end{tabular}

Nota: $\chi^{2} / \mathrm{gl}=$ chi-cuadrado $/$ grados de libertad; CFI = Índice de ajuste comparativo; GFI = Índice de bondad de ajuste; RMR = Raíz cuadrada media residual; RMSEA = Raíz cuadrada media del error de aproximación.

Los resultados señalan que el modelo de cinco factores correlacionados proporciona el mejor ajuste a los datos (Byrne, 2001). A su vez, corrobora los hallazgos obtenidos en el análisis factorial exploratorio dando apoyo empírico a la estructura de cinco factores de la versión en gallego de la escala Cisneros.

\section{Discusión}

El presente estudio tiene como objetivo principal validar la escala Cisneros en la población gallega, analizando la consistencia interna y la estructura factorial de la misma. La adaptación y validación empírica de la escala ha permitido conocer sus propiedades psicométricas y ha ofrecido la oportunidad de avanzar en la evaluación de acoso psicológico en la comunidad gallega.

El análisis factorial de componentes principales aisló nueve factores que explican el $72.03 \%$ de la varianza total, quedando reducidos a cinco factores principales que caracterizan el acoso psicológico en el trabajo en Galicia. El primero, degradación personal, es el que explica un mayor porcentaje de varianza $(40.02 \%)$ e incluye comportamientos tales como las críticas y reproches a cualquier actividad que emprende la víctima, los gestos de menosprecio y humillación pública que buscan desestabilizar a la víctima y provocar que cometa errores en su trabajo, o los rumores malintencionados sobre aspectos personales o, incluso, sobre supuestos errores cometidos en el desempeño de sus tareas. El segundo factor, denominado degradación profesional, incluye comportamientos dirigidos a limitar profesionalmente a la víctima, bien asignándo- para llevar a cabo su tarea. Por último, el quinto factor, denominado ninguneo, aglutina aquellas conductas dirigidas a reducir las posibilidades de la víctima de comunicarse adecuadamente con otros, incluido el propio agresor.

La existencia de un único factor que aglutina un alto porcentaje de varianza explicada $(40.02 \%)$ y el hecho de que la validación en castellano concluya una solución multidimensional de la escala (Fidalgo y Piñuel, 2004), nos ha llevado a realizar un análisis factorial confirmatorio cuyo resultado indica que el modelo de cinco factores correlacionados es el que mejor se ajusta a los datos.

Todas estas conductas han sido señaladas por los investigadores de mobbing y coinciden en cierta forma con el planteamiento teórico sobre la naturaleza de las conductas de mobbing propuesto por Leymann (1996). $\mathrm{Al}$ igual que el trabajo de Leymann, las dimensiones del presente trabajo se refieren a actos que persiguen el descrédito y la humillación personal y profesional de la persona, el aislamiento o ninguneo y el daño de su salud. De hecho, el concepto de mobbing gira en torno a la naturaleza negativa y no deseada de las conductas sufridas por las víctimas, que se ven sometidas a una amplia variedad de comportamientos hostiles relacionados tanto con su vida profesional (por ejemplo, exceso de críticas sobre el trabajo desempeñado o los resultados del mismo, retención de información necesaria para el desempeño de las tareas, aislamiento social, etc.) como con su vida personal (por ejemplo, ataques contra la vida privada, rumores, insultos, etc.), llegando en ocasiones a ser objeto de amenazas y de actos de violencia física (Einarsen, 2000). 
Esta solución factorial es bastante similar, tanto en lo que respecta al porcentaje de varianza explicada como al número de factores, a su contenido y a su fiabilidad, a la encontrada en otras investigaciones sobre el acoso psicológico (Fornés et al., 2008; Leymann, 1990; Meseguer et al., 2007; Niedl, 1995).

$\mathrm{Al}$ igual que en este estudio, las soluciones factoriales de la mayoría de las escalas de mobbing señalan la existencia de un factor que aglutina un porcentaje elevado de la varianza. En nuestro caso se trata del factor denominado degradación personal que aglutina 17 ítems y explica el $40 \%$ de la varianza. El análisis factorial del LIPT realizado por Niedl (1995) señala que el primer factor, ataques a la integridad personal, aglutina un total de 13 ítems, explicando un $36.5 \%$ de la varianza. Un caso similar es la solución factorial propuesta por Einarsen y Raknes (1997) para el NAQ, cuyo primer factor (ataques personales) formado por 9 ítems explica el $31.7 \%$ de la varianza. Por su parte, Fornés et al. (2008) obtiene porcentajes similares de varianza explicada por un factor formado por 12 ítems y denominado humillación y rechazo personal $(39.3 \%)$, mientras que el primer factor obtenido por Meseguer et al. (2007) explica 30.98\% de la varianza, saturando en él 8 ítems relacionados con ataques de tipo personal. Incluso, algunos autores proponen soluciones factoriales en las que el primer factor explica un 50\% de la varianza (Moreno-Jiménez et al., 2008). Cabe señalar que en todos los casos citados, el factor que explica un mayor porcentaje de varianza se refiere a conductas de tipo personal. Esta distinción en función del objeto del ataque ha sido puesta de manifiesto por Einarsen y Hoel (2001) al plantear en su estudio dos categorías de conductas, una formada por ataques hacia la persona (personal bullying) y otra relacionada con el desempeño del trabajo (work-related bullying).

En cuanto al número de factores encontrados, los resultados indican la existencia de cinco factores principales que explican un porcentaje total de varianza del $60.3 \%$. El análisis factorial propuesto por Fornés et al. (2008) para el HPT señala también la existencia de cinco factores (Humillación y rechazo personal; Desprestigio profesional; Rechazo profesional y violación de la intimidad; Degradación profesional; Ninguneo-aislamiento profesional) que aglutinan un total de 35 ítems y que explican un 50.5\% de la varianza. De igual modo, Meseguer et al. (2007) propone una solución factorial del NAQ compuesta por cinco factores que explican el 54\% de la varianza. Sin embargo, García-Izquierdo et al. (2004) eleva al $66.7 \%$ el porcentaje de varianza explicada a partir de cuatro factores del NAQ.

Por último, respecto a la fiabilidad de toda la escala, los resultados muestran una elevada fiabilidad del instrumento $(\alpha=.96)$, similar a la encontrada por Fidalgo y Piñuel (2004) por el método de las dos mitades $(\alpha=.96)$ y por el coeficiente alfa de Cronbach $(\alpha=.97)$. Además, se ha obtenido una fiabilidad adecuada para cada uno de los factores con coeficientes alfa que varían de .75 (Ninguneo) a .96 (Degradación personal), situándose en línea con los trabajos mencionados anteriormente cuyos coeficientes alfa varían entre .83 (Ninguneo-aislamiento profesional) y .92 (Humillación y rechazo personal) en el caso de Fornés et al. (2008), y entre .78 (Conductas discriminatorias asociadas al género y a la edad) y .88 (Conductas hostiles que afectan al entorno social) en el caso de García-Izquierdo et al. (2004).

En definitiva, la versión en gallego de la escala Cisneros presenta buenas propiedades psicométricas, permite evaluar el mobbing y proporciona un instrumento adecuado para detectar diferentes formas de acoso psicológico en el trabajo e identificar a los responsables de las mismas.

\section{Extended Summary}

The CISNEROS scales measures the incidence of mobbing in the work environment. Taking into account that it is one of the first instrument for measuring mobbing that has been validated for Spain and that there exists no instrument translated into Galician and validated for Galicia, the aim of this study is to validate the CISNEROS scale in the Galician population, analysing the internal consistency and the factorial structure of the same.

\section{Method}

\section{Participants}

We used a sample of 384 workers above the age of 18 belonging to the Galician Autonomous region -rep- resentative of the active population $(e= \pm 5.0 \%) ; 218$ men $(56.8 \%)$ and 166 women (43.2\%), with an average age of 33.21 years old $(D T=10.62)$.

The most relevant demographical and work characteristics are detailed in Table 2.

\section{Instruments}

The CISNEROS scale (Fidalgo \& Piñuel, 2004), objectivises 43 behaviours of mobbing in order to indicate the frequency with which each one is suffered on a scale which goes from 0 (never) to 6 (every day). At the same time it allows us to identify where the mobbing of each behaviour comes from, distinguishing between psychological mobbing which comes from bosses, colleagues or subordinates. It offers three 
harassment indexes: the total number of harassment strategies (NEAP), the global index of psychological harassment (IGAP) and the average index of the intensity of harassment strategies (IMAP). The scale also includes an additional item of dichotomy response yes/no (item 44) which sums up the subjective perception of the subject in order to consider a case as mobbing. With respect to the psychometric properties of the original scale we should like to point out that Fidalgo and Piñuel found a reliability (Cronbach alpha) of .97 .

\section{Procedure}

Following the guidelines suggested by CarreteroDios and Pérez (2005) we developed the adaptation of the CISNEROS scale to Galician in three phases: (1) translation, (2) pilot test, and (3) validation.

The firs step was to translate the scale into Galician for which we had the help of the Servicio de Normalización Lingüistica of the University of Compostela. Once translated into Galician, the scale was then translated back into Spanish to check that the original meaning of each item had been respected. This translation procedure converges with the framework set out by Brislin (1986) in respect to the equivalence of translations.

The next step consisted in carrying out a pilot test to check the linguistic comprehension and language of the questionnaire. The test was carried out using a nonclinical sample made up of students from different University campuses in Galicia, with the aim of testing for comprehension in geographical regions with very different registers of Galician. Finally the questionnaire was administered during the months of September and October 2007 to the chosen sample after obtaining verbal agreement from those interested in taking part in the study, with the aim of validating the test in the Galician population, and whose results are shown in this work. The analysis of the date was carried out using the statistical packet (SPSS 15.0).

\section{Results}

We used the Main Components Method with Varymax rotation for the extraction of the factors and we retained those factors with eigenvalue or self value greater than 1.00 (Kaiser, 1960). To assign the items to the factors we took into consideration factorial loads equal or greater than I.40I (Nunnally, 1978). Once the factors had been obtained their reliability was calculated using the Cronbach alpha coefficient.

Nine factors were obtained with a self value above 1 , which explains $72.03 \%$ of the total variance. In Table 3 we show the factorial saturations of the five first factors which in total explain $60.31 \%$ of the total variance of the test. They were given names based on the predominant content of the items which made them up and on existing literature. The final four factors are not included as they were considered residual since they only contain one or two items and explained a low percentage of variance. The list of factors is as follows:

- Factor 1: Personal degradation. This alone explains $40.02 \%$ of the variance. It is made up of 17 items with saturations ranging from .46 to .84 and include a wide spectrum of harassment behaviours such as criticism and reproaches (items 17, 18 and 32), belittling (items 15,19,24 and 40), provocations in order to force errors (items 23, 42 and 41), threats (items 20 and 27), shouting (item 29), discrimination (item 5) and other measures aimed at damaging one's image (item 35), distorting communication (item 22) and social isolation of the employee (item 21).

- Factor 2: Professional degradation. This explains $6.56 \%$ of the variance. It is made up of seven items with saturations which go from .41 to .79 . Its content refers to behaviour leading towards to professional limitation through the use of reduction of deadlines (item 37), undue pressure (item 36), treatment below the level of skill (items 38, 8 and 9) undervaluing of effort (item 39) and withholding information (item 33).

- Factor 3: Personal humiliation. This is made up of four items which explain $5.63 \%$ of the variance. All the saturations are above .66 and content refers to behaviour aimed at humiliating the person with continual criticism of his/her personal life (item 26), ridicule (item 31), being laughed at (item 25), even going as far as physical dominance (item 30 ).

- Factor 4: Deliberate exposure to risks. This explains $4.10 \%$ of the variance. It is made up of four items with saturations between .59 and .78 and its content refers to deliberate and badly willed conduct such as the apportioning of dangerous tasks (item11), impeding the use of safety procedures (item 12), overburdening with work (item 10) and limiting professional advancement (item 34 ).

- Factor 5. Ninguneo. This consists of three items which explain $3.99 \%$ of the variance, with saturations above .67. The content of these items refers to conduct such as ignoring (item 2), continual interruption (item 3) and restriction of communication (item 1).

In table 4 we show the descriptive statistics, reliability and correlations corresponding to the five factors which make up the scale for the sample total.

Although exploratory factor analysis shows a multidimensional structure, the detailed analysis of the factors extracted suggests that the scale could have only one factor, because the first factor (personal degrada- 
tion) groups 17 items and explains $40 \%$ of the total variance of the scale. In order to clarify the factor structure of the scale we carried out a confirmatory factor analysis with the aim of comparing three different models (one factor, five independent factors and five correlated factors). Table 5 summarizes the fit of the three models compared. The results indicate that the correlated five-factor model provided the best fit to the data (Byrne, 2001).

\section{Discussion}

The main components factor analysis isolated nine factors which explain $72.03 \%$ of the total variance resulting in five main factors which characterize psychological harassment at work in Galicia (personal degradation, professional degradation, personal humiliation, deliberate exposure to risks and ninguneo). Also, we compare three models fit using confirmatory factor analysis (one factor, five independent factors and five correlated factors). The results indicate that the correlated five-factor model provided the best fit to the data, giving empirical support to the five-factor structure obtained in the exploratory factor analysis.

This factorial solution is quite similar, both in relation to the percentage of variance explained and to the number of factors, to their content and reliability to that which is to be found in other research into psychological harassment (Fornés et al., 2008; Leymann, 1990; Meseguer et al., 2007; Niedl, 1995).

Just as in this study, the factorial solutions in the majority of mobbing scales point to the existence of a factor which brings together a high percentage of the variance. In our case it is the factor denominated personal degradation which brings together 17 items and explains $40 \%$ of the variance. It is important to point out that in all the cases mentioned, the factor that explains a large percentage of variance refers to conducts of a personal type. This distinction in the function of the object of the attack was pointed out by Einarsen and Hoel (2001) who put forward in their study two categories of conduct, one made up of attacks on the person (personal bullying) and another related to the carrying out of work (work-related bullying)

As for the number of factors found the results indicate existence of five factors which explain a total variance percentage of $60.3 \%$. The factorial analysis put forward by Fornes et al. (2008) for the HPT also points out the existence of five factors (humiliation and personal rejection; professional belittlement; professional rejection and invasion of privacy; professional degradation; ninguneo-professional isolation) which brings together a total of 35 items and explain $50.5 \%$ of the variance. Likewise Meseguer et al. (2007) propose a factorial solution of NAQ made up of five factors which explain 54\% of the variance. However, GarcíaIzquierdo et al. (2004) raises the percentage of variance explained from four factors of the NAQ to $66.7 \%$.

With regard to the reliability of the whole scale, the results show a high instrument reliability (alpha .96) similar to that found by Fidalgo and Piñuel (2004) using the two halves method (alpha .96) and through the Cronbach alpha coefficient alpha .97). We also obtain a suitable reliability for each one of the factors with alpha coefficients which range from .75 (ninguneo) to .96 (personal degradation), in line with the works mentioned before whose alpha coefficients vary between .83 (ninguneo-professional isolation) and .92 (humiliation and personal rejection) in the case of Fornés et al. (2008), and between .78 (discriminatory conduct associated to gender and age) and .88 (hostile behaviour which affects social environment) in the case of García-Izquierdo et al. (2004).

In short, the Galician version of the CISNEROS scale presents good psychometric properties, allows us to evaluate mobbing and provides a suitable instrument for detecting different forms of psychological bullying in the workplace.

\section{Referencias}

Agervold, M. (2007). Bullying at work: A discussion of definitions and psevaleme, based on an empirical study. Seanelinavian Journal of Psychology, 48, 161-172.

Arbuckle, J. L. (2005). AMOS 6.0 User's Guide. Chicago, IL: SPSS Inc.

Archer, D. (1999). Exploring bullying culture in the paramilitary organization. International Journal of Manpower, 20, 94-105.

Björkqvist, K., Österman, K., y Hjelt-Bäck, M. (1994). Aggression among university employees. Aggressive Behavior, 20, 173-184.

Boada, J., De Diego, R., y Vigil, A. (2003). Mobbing: Análisis de las propiedades psicométricas y estructura factorial de cuatro escalas (MOBB-90; MobbCF-21; MobbCG-15 \& MobbCS-28). Revista de Encuentros de Psicología Social, 1, 26-31.

Brislin, R. W. (1986). The wording and translation of Research Instruments. En W. J. Lonner y J.W. Berry (Eds.), Field Methods in Cross-Cultural Research (pp. 137-164). Beverly Hills: Sage.

Byrne, B. (2001). Structural equation modelling with AMOS, EQS, and LISREL: Comparative approaches to testing for the factorial validity of a measuring instrument. International Journal of Testing, 1, 55-86.

Carretero-Dios, H. y Pérez, C. (2005). Normas para el desarrollo y revisión de estudios instrumentales. International Journal of Clinical and Health Psychology, 5, 521-551.

Cortina, L. M., Magley, V. J., Williams, J. H. y Langhout, R. D. (2001). Incivility in the workplace: Incidence and impact. Journal of Occupational Health Psychology, 6, 64-80. 
Coyne, I., Seigne, E. y Randall, P. (2000). Predicting workplace victim status from personality. European Journal of Work and Organizational Psychology, 9, 335-349.

Einarsen, S. (2000). Harassment and bullying at work: A review of the scandinavian approach. Aggression and Violent Behavior: A Review Journal, 5, 371-401.

Einarsen, S. y Hoel, H. (2001, mayo). The Negative Acts Questionnaire: Development, validation and revision of a measure of bullying at work. Ponencia presentada al $10^{\text {th }}$ European Congress on Work and Organizational Psychology, Praga, República Checa.

Einarsen, S. y Hoel, H. (2006). Measuring bullying and harassment in the workplace. Development and validity of the revised Negative Acts Questionaire. A manual. Bergen: University of Bergen

Einarsen, S. y Raknes, B. I. (1997). Harassment at work and the victimization of men. Violence and Victims, 12, 247-263.

Einarsen, S., Raknes, B. J. y Matthiesen, S. B. (1994). Bullying and harassment at work and their relationships to work environment quality. An exploratory study. European Work and Organizational Psychologist, 4, 381401.

Fidalgo, A. M. y Piñuel, I. (2004). La escala Cisneros como herramienta de valoración del mobbing. Psicothema, 16, 615-624.

Fornés, J., Martínez-Abascal, M. A. y García de la Banda, G. (2008). Análisis factorial del Cuestionario de Hostigamiento Psicológico en el Trabajo en profesionales de Enfermería. Internacional Journal of Clinical and Health Psychology, 8, 267-383.

García, E., Gallo, P. y Miranda, R. (1998). Bondad de ajuste en el análisis factorial confirmatorio. Psicothema, 10, 717-724.

García-Izquierdo, M., Llor, B., Sáez, M. C., Ruiz, J. A., Blanco, J. R. y Campillo, M. J. (2004). Evaluación del acoso psicológico en el trabajo: El NAQ-RE. Revisión de la adaptación española. Comunicación oral presentada al VII European Conference on Psychological Assessment, Málaga, España.

González de Rivera, J. L. y Rodríguez-Abuín, M. (2003). Cuestionario de estrategias de acoso psicológico: El LIPT-60 (Leymann Inventory of Psychological Terrorization) en versión española. Psiquis, 24, 59-66.

Gross, C. (2002, septiembre). Social conflicts and bulllying at work: First results of a diary study. Ponencia presentada a la International Conference on Bullying and Harassment at work, Londres, Reino Unido.

Hair, J., Anderson, R., Tatham, R. y Black, W. (1999). Análisis multivariante ( $5^{\mathrm{a}}$ ed.). Madrid, España: Prentice Hall.

Kaiser, H. F. (1960). The application of electronic computers to analysis factorial. Educational and Psychological Measurement, 20, 141-151.

Leymann, H. (1990). Handbook for anviindning av LIPTformuliiret for kartliiggning av risker for psykiskt void I arbetsmiljon [Manual of the LIPT questionnaire for assessing the risk of psychological violence at work]. Estocolmo, Suecia: Violen.
Leymann, H. (1996). The content and development of mobbing at work. European Journal of Work and Organizational Psychology, 5, 165-184.

Liefooghe, A. y Olafsson, R. (1999). "Scientists" and "amateurs" mapping the bullying domain. International Journal of Manpower, 20, 39-49.

Liefooghe, A. y Mackenzie Davey, K. (2001). Accounts of workplace bullying: The role of the organization. European Journal of Work and Organizational Psychology, 10, 375-392.

Liefooghe, A. y Mackenzie Davey, K. (2003). Explaining bullying at work. Why should we listen to employee accounts? En S. Einarsen, H. Hoel, D. Zapf y C. L. Cooper (Eds.), Bullying and emotional abuse in the workplace. International perspectives in research and practise (pp. 219-244). Londres, Reino Unido: Taylor \& Francis Books Ltd.

Martin, R. J. y Hine, D. W. (2005). The development and validation of the Uncivil Workplace Behavior Questionnaire. Journal of Occupational Health Psychology, 10, 477-490.

Matthiesen, S., Aasen, E., Holst, G., Wie, K. y Einarsen, S. (2003). The escalation of conflict: a case study of bullying at work. International Journal of Management and Decision Making, 4, 96-112.

Meseguer, M., Soler, M. I., Sáez, M. C. y García-Izquierdo, M. (2007). Incidencia, componentes y origen del mobbing en el trabajo en el sector hortofrutícola. Anales de Psicología, 23, 92-100.

Montero, I. y León, O. G. (2007). A guide for naming research studies in Psychology. International Journal of Clinical and Health Psychology, 7, 847-862.

Moreno-Jiménez, B., Rodríguez-Muñoz, A., Martínez, M. y Gálvez, M. (2007). Assessing workplace bullying: Spanish validation of a reduced version of Negative Acts Questionnaire. The Spanish Journal of Psychology, 10, 449-457.

Moreno-Jiménez, B., Rodríguez-Muñoz, A., Morante, M. E., Garrosa, E., Rodríguez-Carvajal, R. y Díaz-Gracia, L. (2008). Evaluación del acoso psicológico en el trabajo: Desarrollo y estudio exploratorio de una escala de medida. Universitas Psychologica, 7, 335-345.

Niedl, K. (1995). Mobbing/Bullying am Arbeitsplatz. Eine empirische Analyse zum Phänomen sowie zu personalwirtschaftlich relevanten Effekten von systematischen Feindseligkeiten [Mobbing/bullying at work: An empirical analysis of the phenomenon and of the effects of systematic harassment on human resource management]. München, Alemania: Hampp.

Nunnaly, N. C. (1978). Psychometric theory. Nueva York: McGraw-Hill.

Parent-Thirion, A., Fernández-Macías, E., Hurley, J. y Vermeylen, G. (2007). Fourth European Working Conditions Survey. Recuperado el 22 de febrero de 2007, de http://www.eurofound.eu.int/pubdocs/2006/98/en/2/ef 0698en.pdf

Piñuel, I. y Oñate, A. (2006). La evaluación y diagnóstico del mobbing o acoso psicológico en la organización. El 
Barómetro Cisneros. Revista de Psicología del Trabajo y de las Organizaciones, 22, 309-332.

Rospenda, K. M. y Richman, J. A. (2004). The factor structure of generalized workplace harassment. Violence and Victims, 19, 221-238.

Van Dick, R. y Wagner, U. (2001). Stress and strain in teaching. A structural equation approach. British Journal of Educational psychology, 71, 243-259.
Zapf, D. (1999). Mobbing in organisationen. Ein überblick zum stand der forschung [Mobbing in organisations. A state of the art research review]. Zeitschrift für Arbeits- \& Organisationspsychologie, 43, 1-25.

Zapf, D., Knorz, C. y Kulla, M. (1996). On the relationships between mobbing factors, and job content, social work environment and health outcomes. European Journal of Work and Organizational Psychology, 5, 215-238.

Manuscrito Recibido: 01/07/2009

Revisión recibida: 12/01/2009

Aceptado: 12/11/2009 